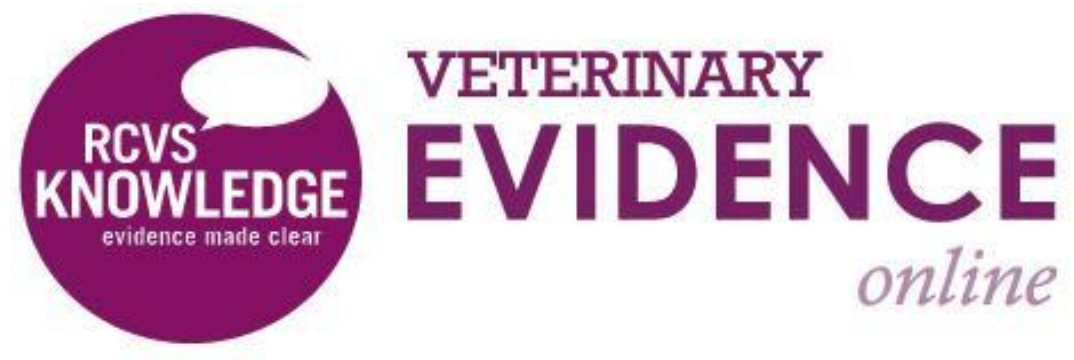

\title{
Evaluation of Factors Influencing Thigh Circumference Measurement in Dogs
}

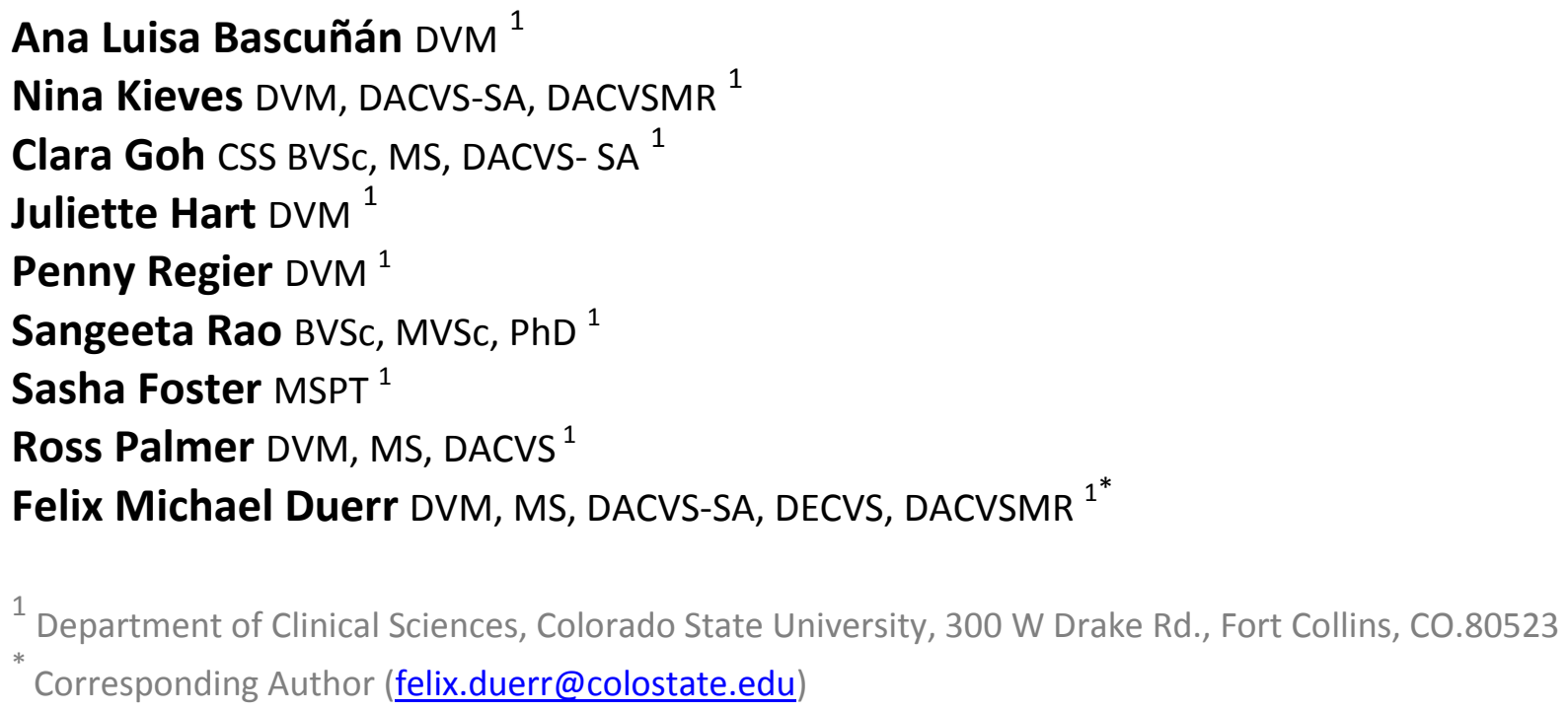

ISSN: 2396-9776

Published: 05 May 2016

in: Vol 1, Issue 2

DOI: http://dx.doi.org/10.18849/ve.v1i2.33

Reviewed by: Wanda Gordon-Evans (DVM, PhD, DACVS DACVSMR) 
Objective: To evaluate inter- and intra-observer variability, influence of hair clipping and laser guidance on canine thigh circumference (TC) measurements amongst observers.

Background: It was our goal to further study the reliability of canine TC measurements as currently performed. For this purpose we designed a cadaveric model that allows for controlled inflation of the thigh resembling increase of muscle mass. We also investigated the impact of novel technologies (laser guidance) and hair clipping on TC measurements in this model.

Evidentiary value: Phase 1 cadaveric study - five long-haired, large breed canine cadavers; Phase 2 clinical study - eight clinically healthy Golden Retrievers. This study should impact clinical research and practice.

Methods: Phase 1 - Canine cadaveric thigh girth was manually expanded to three different levels using a custom, submuscular inflation system before and after hair clipping; Phase 2 - TC of Golden Retrievers was measured with and without laser guidance. TC measurements for both phases were performed by four observers in triplicate resulting in a total of 552 measurements.

Results: Phase 1 - TC measurements before and after hair clipping were significantly different $(3.44 \mathrm{~cm}$ difference, $p<0.001)$. Overall inter-observer and intra-observer variability were $2.26 \pm 1.18 \mathrm{~cm}$ and $0.90 \pm 0.61 \mathrm{~cm}$, respectively. Phase 2 - Laser guidance nominally improved inter-observer variability $(3.34 \pm 1.09 \mathrm{~cm}$ versus 4.78 $\pm 2.60 \mathrm{~cm})$ but did not affect intra-observer variability $(1.14 \pm 0.66 \mathrm{~cm}$ versus $1.13 \pm 0.77 \mathrm{~cm})$.

Conclusion: TC measurement is a low fidelity outcome measure with a large inter- and intra-observer variability even under controlled conditions in a cadaveric setting. Current methods of canine TC measurement may not produce a valid outcome measurement. If utilised, hair coat clipping status should be considered and an intra-observer variability of at least $1 \mathrm{~cm}$ should be assumed when comparing repeated TC measurements. Laser guidance may be helpful to nominally reduce inter-observer variability in settings with multiple observers. Further investigation of alternative methods for canine TC measurement should be pursued.

Application: This information should be considered by everyone utilizing TC measurements as an outcome assessment for clinical or research purposes.

INTRODUCTION

Assessment of muscle mass in canine patients is a commonly used subjective and objective outcome measure for evaluation of musculoskeletal disease (Monk et al., 2006, Moeller et al., 2010, Eskelinen et al., 2012, Gordon-Evans et al., 2011, Kalis et al., 2012, Innes and Barr, 1998). Definitive measurement of muscle mass involves advanced imaging, making it impractical and cost prohibitive in most clinical cases. Limb circumference measurement with a tape measure is frequently used in both human and veterinary patients as an indirect measure of changes in muscle mass over time (Doxey, 1987a, Doxey, 1987b, Jarvela et al., 2002, Thomaes et al., 2012).

Practical and affordable means of outcome measurement are critical to the veterinary field. As an example, cranial cruciate ligament disease (CCLD) is a common orthopaedic disease in the canine patient with substantial on-going research evaluating treatment outcomes (Kim et al., 2008),(Slocum and Slocum, 1993). TC has been used to evaluate symmetry in muscle mass between pelvic limbs and hence is often used as an objective evaluation of response to treatment of CCLD (Gordon-Evans et al., 2010, Gordon-Evans et al., 2011, 
Gordon-Evans et al., 2013, Moeller et al., 2010). Unfortunately previous studies evaluating canine TC measurement using various tape measures have shown significant inter- and intra-observer variability (Smith et al., 2013, Baker et al., 2010). Use of a spring-tensioned device (GT) is recommended to decrease variability in the force used by the observer when tensioning the tape measure around the limb (Millis et al., 1999, Millis, 2004). However, this technology is reportedly only reliable for measurements of the proximal antebrachium in canines and its validity for thigh circumference measurement has been questioned (Smith et al., 2013). Without reliable methods for TC measurement, this assessment may give an incomplete or erroneous evaluation of response to therapy and intervention.

The reason for the previously reported poor reliability of canine TC measurement is unclear. Potential variables include hair coat length, variation in the site of measurement due to a lack of clearly identifiable landmarks on the limb, limb position, and dog movement. To improve reliability of measurements in human subjects, several devices have been created that control height and angle of the measurement along the limb (Berard and Zuccarelli, 2000, Berard et al., 1998, Berard et al., 2002, Soderberg et al., 1996). However, these devices are difficult to apply to the canine patient due to differences in anatomy. The purpose of this study was to evaluate the impact these potential variables play in TC measurement of the canine. To eliminate the need to rely on palpation of anatomic landmarks, the authors of the present study used a device that projected a laser line across the canine limb, guiding placement of the measuring tape across the thigh. To eliminate dog movement as a factor, we developed a cadaveric model affixing the limb in space while measuring TC. To evaluate hair coat as a factor, cadavers were measured pre- and post-hair clipping.

Our specific aims were to determine if hair coat clipping affects TC measurement, to determine the intra- and inter-observer variability of TC under constant conditions using an affixed limb, and to evaluate the effect of laser guidance on inter- and intra-observer variability of TC measurements in a clinical setting. We hypothesised that hair coat clipping would not significantly affect TC measurements made with a GT, and that using a laser guidance device would decrease both intra- and inter-observer variability of TC measurements.

METHODS \& MATERIALS

This study was performed in two phases: First, to evaluate the effect of hair coat length (pre- and postclipping) across a range of muscle masses and to determine the inter- and intra-observer variability of TC measurement under constant conditions, cadaveric limbs were measured in a fixed position. Second, to evaluate the effect of laser guidance on TC measurement, live dogs were measured with and without a device that projects a laser line across the canine limb.

Phase 1: Cadaveric study - Cadavers of long-haired, large breed canines $(n=5)$ euthanized for reasons unrelated to the study were utilised. A custom, submuscular thigh expansion system was developed to simulate incrementally increasing thigh muscle mass in a cadaveric model. Inflation devices were created by attaching surgical gloves (Perry Orthopaedic, size 7.5, Red Bank, NJ, USA) to red rubber catheters (20 French, Tyco Healthcare group, Mansfield, MA, USA) with a Finger Trap suture pattern. A standard cranio-lateral approach to the femur of the right hind limb of each cadaver was performed, and one inflation device was inserted in between the femur and quadriceps muscle group, and a second device was inserted between the adductor and biceps femoris muscle bellies. Anatomic closure was performed to secure the inflation devices in place under the muscle bellies. The distal limb was secured to a wooden block at the metatarsus to ensure no limb movement occurred between measurements. The cadavers were positioned in lateral recumbency for TC measurement. A 60cc syringe (Monoject, Covidien, Mansfield, MA, USA) was attached to each of the red rubber catheters, and each device was inflated or deflated with water to one of three predetermined volumes $(0 \mathrm{ml}, 60 \mathrm{ml}$, or $120 \mathrm{ml}$ total volume split evenly between the two inflation devices) in random order. Observers were blinded to the inflation volume. After the measurements were made, the water was removed from the inflation devices and confirmed to be the same total volume $(120 \mathrm{ml})$ to detect any leakage within the system. 
Each of the observers was instructed in how to measure TC using the GT (Country Technology, Inc. Model \#67019, Gays Mills, WI) and given time to practice making measurements until the procedure was routine. Observers then measured TC of each limb three times at each level of inflation before and after clipping of fur. Fur was clipped in surgical fashion (to the skin) with a number 40 blade in a complete ring around the thigh. To reduce variability in position of the GT on the lateral aspect of the limb between repeated measurements and between observers, a laser line device (Cross Line Self Leveling Laser, Model \# GLL2-10, Bosch, USA) was positioned to project a line at the estimated midpoint between the palpated anatomic landmarks (greater trochanter and the lateral femoral condyle) to guide measurement in all cadavers. The laser device was selfleveling to ensure consistent angle of projection of the beam. Cadavers remained in the same position throughout data collection to avoid any variability in the positioning of the laser guidance line while performing serial measurements. The GT used for all measurements was 'blinded' to observers by covering the outward facing measurement scale with black electrical tape. An independent observer recorded measurements by carefully pinching the tape without imparting any movement and then rotating it to visualise the inward facing scale. The observer recording the blinded measurements was the same throughout the study to avoid variability incurred while pinching and rotating the tape. The GT was completely removed from around the thigh between each consecutive measurement.

Phase 2: Live dog study - Live dogs were used to evaluate TC measurements made using the GT and the laser line device in a clinical setting. The same trained observers who participated in the cadaveric portion of the study measured the TC of healthy Golden Retrievers $(n=8)$ using the blinded GT with and without the laser device for guidance.

For non-laser guided measurements, TC was measured as previously described (midway between the palpated greater trochanter and lateral femoral condyle) with the dog in normal weight bearing position (Smith et al.,

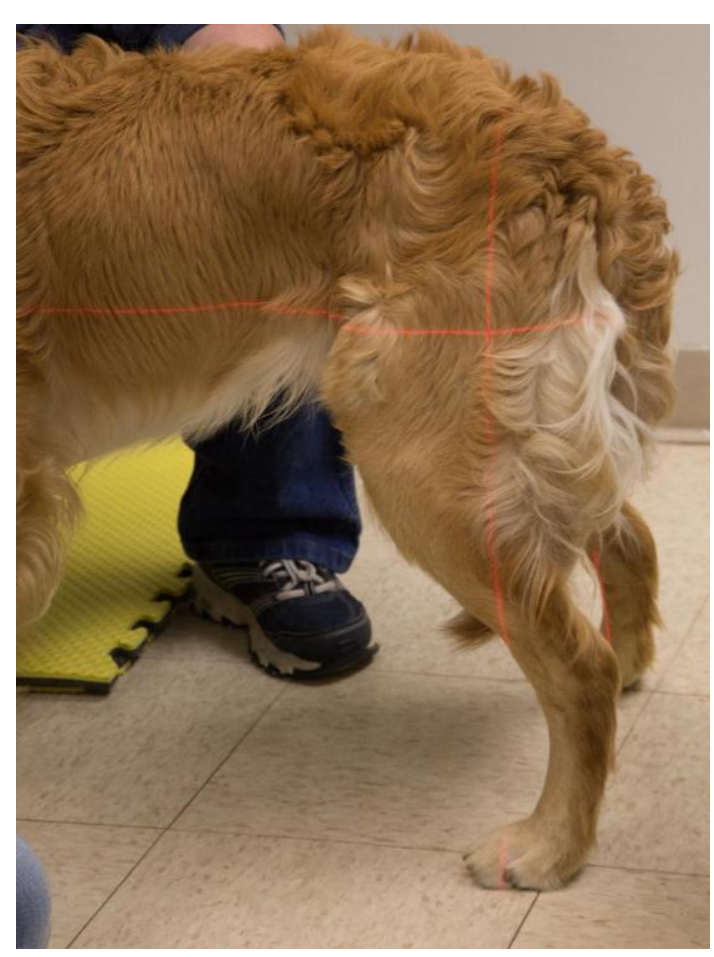
2013). For laser-guided TC measurements, dogs were positioned in a normal weight bearing, square stance on a location marked with an ' $X$ ' on the floor. This was done to decrease the variability in distance from the laser device. The laser level was placed on a tripod and positioned such that it projected at mid-thigh level on each dog (Figure 1). The height of the laser level beam on the wall was recorded for each dog so that the same level of measurement could be replicated between observers and measurements. Each observer measured the left pelvic limb three times with and without laser guidance in random order and the GT was removed from around the thigh between each consecutive measurement. When laser guidance was used, TC measurements were performed along the displayed laser line.

Figure 1: This image depicts the left hind limb of a Golden Retriever with the laser line device positioned midway between the greater trochanter and the lateral femoral condyle to guide measurement

Statistical analysis - The data analyses were performed using SAS v9.3 (SAS Institute Inc., Cary, NC, USA). Variability was defined as the mean range in measurements performed under the same circumstance (i.e. the same inflation level or dog); thus a value of 0 would indicate an ideal test with no inherent variability. Difference was defined as the mean difference between measurements performed under different circumstances (i.e. different inflation levels or clipped vs. intact hair coat). The data in both live and cadaver dogs were evaluated for normality assumptions. The data on live dogs were analysed using a bivariable linear 
regression analysis to compare the difference in mean circumference between laser guided and non-laser guided measurements. A bivariable linear regression was also used to evaluate the differences in measurement among the observers. Multiple measurements by the same observer on an individual dog were included in the analysis to account for the variability around the mean from repeated observations. The data on cadaver dogs were evaluated using a multivariable linear regression analysis. Repeated measures on the same dog were taken into account in the analysis. TC difference was calculated using repeated observations within each dog from all observers. A $p$-value $<0.05$ was considered for determining statistical significance.

\section{RESULTS}

Phase 1: Cadaveric data - Five long-haired, large breed canine cadavers were used (3 Golden Retrievers, 1 St. Bernard, and 1 Malamute). A total of 360 measurements were performed. Inter-observer variability in TC measurement when all inflation levels were combined was $2.26 \pm 1.18 \mathrm{~cm}$. Intra-observer variability in TC measurement for all 4 observers when all inflation levels were combined was $0.90 \pm 0.61 \mathrm{~cm}$ (see Table 1). Mean TC measurement was significantly different between the three inflation levels of the submuscular device (see Table 2). TC measurements made on long-haired (unclipped) limbs at all inflation levels were significantly different than those made on the same limbs after clipping the hair (see Table 2). Inter-observer variability did not improve with clipping of hair coat (see Table 1).

Phase 2: Live dog data - Eight clinically healthy Golden Retrievers were used. A total of 192 measurements were performed. For measurements made with laser guidance, TC measurements between one pair of observers (Observers 1 and 3) differed significantly. In the non-laser guided group three observer comparison pairs were significantly different in their TC measurements (see Table 3). The inter-observer variability was smaller in the laser guided group compared to the non-laser guided group $(3.34 \pm 1.09 \mathrm{~cm}$ compared to 4.78 $\pm 2.60 \mathrm{~cm}$ ). However, no statistically significant difference in either intra- or inter-observer variability was found between laser guided and non-laser guided measurement groups (see Table 4).

\section{DISCUSSION}

The use of TC measurement is often cited as an objective measure of response to treatment of various pelvic limb injuries and diseases. To our knowledge, no peer-reviewed studies have evaluated the effect of hair coat clipping on TC measurement, or the use of laser guidance to decrease variability of TC measurement. Furthermore, no peer-reviewed studies have evaluated the inter- and intra-observer variability of TC under constant conditions. We developed a cadaveric model that allowed us to mimic progressively increasing muscle mass while eliminating patient motion. This model also allowed us to evaluate hair clipping as the only variable in actual thigh circumference. In the live dog study we attempted to improve consistency of placement of the GT for TC measurement by utilising laser guidance. The results of our study show that TC measurement is a low fidelity outcome measure that should be utilised and interpreted cautiously in clinical practice.

Even under highly controlled conditions using affixed cadaveric pelvic limbs, large and clinically significant inter- and intra-observer variability was demonstrated. We found the difference between TC measurements at each of the three inflation levels of the submuscular expansion device to be statistically significant with $\sim 1 \mathrm{~cm}$ increase in TC per inflation level, suggesting that this custom model is suitable for future application. The difference seen was similar to previous reports using in vivo models of muscle hypertrophy (Monk et al., 2006, Gordon-Evans et al., 2010, Gordon-Evans et al., 2011, Gordon-Evans et al., 2013). When considering this value, 
an inter-observer variability in measurement of more than $2 \mathrm{~cm}$ demonstrates that the current guidelines for TC measurement are not sufficient to provide reproducible results between observers. This is consistent with previous studies that demonstrated poor intra- and inter-observer reliability in canine TC measurement in a clinical setting (Smith et al., 2013, Baker et al., 2010): Baker et al. reported an intra-observer variability of $0.57 \mathrm{~cm}$ and an inter-observer variability of $1.51 \mathrm{~cm}$ when measuring canine TC with the GT (Baker et al., 2010). Smith et al. demonstrated poor reliability in canine TC measurement using intra-class correlation coefficients (ICC), with less than 0.59 considered unreliable. The intra-observer ICCs ranged from 0.29-0.60, while the inter-observer ICCs ranged from 0.23-0.43 (Smith et al., 2013).

Previously published studies have attributed variability in measurement to factors such as limb position, dog movement, and site of measurement along the limb (Smith et al., 2013, Baker et al., 2010). The results of our study indicate that in addition to these factors, the technique itself is responsible for this poor reliability. Despite controlling these factors in the cadaveric portion of this study, variability still existed both within and between observers. This suggests that an unidentified variable may affect measurements of TC with the GT. Possible causes of this could be inconsistent placement of the GT on the medial aspect of the thigh, slipping of the GT given the conical shape of the thigh musculature, or differences in clinical experience and skill with the GT. All observers were trained to use the GT prior to data collection; however differences in comfort with or prior exposure to the GT may have contributed to poor reliability in measurement. Given that the measured difference in TC between minimum and maximum inflation levels of the submuscular expansion device was less than $2 \mathrm{~cm}$, an intra-observer variability of almost $1 \mathrm{~cm}$ and an inter-observer variability of over $2 \mathrm{~cm}$ calls to question the ability of the GT to detect true changes in TC even when utilised by a trained observer. Though the GT has been validated in previous studies for the measurement of TC on a single occasion, no study has confirmed the sensitivity of the GT to changes in canine TC while also accounting for the inherent variability in measurement (Baker et al., 2010, Millis, 2004). Furthermore, the clinical significance of changes in TC is not well established in the literature. Moeller et al. demonstrated that 5 years after unilateral tibial plateau leveling osteotomy, dogs had significantly smaller TCs on the operated limb compared to the non-operated limb, but that no subjective lameness was associated with this difference.(Moeller et al., 2010) Given the results from the present study, it is possible that the large standard deviation and minimal difference between groups in the Moeller study $(39.5 \pm 5.5 \mathrm{~cm}$ vs. $40.1 \pm 5.6 \mathrm{~cm})$ resulted in a type-I statistical error.

We found that hair clipping affects measurement of TC performed with a GT. This finding is of particular importance when evaluating TC after surgical procedures or other procedures that require hair clipping. Millis et al. studied variables affecting limb circumference measurement with the GT (Millis et al., 1999, Millis, 2004). It was reported in the non-peer reviewed literature that hair coat length had minimal effect on measurement, however only short-haired dogs were used in that study (Millis, 2004, Millis et al., 1999). In the current study, hair coat status significantly affected measurements with the GT. This is most likely due to the tensioning device being designed for use in humans rather than canines. Since humans have little variation in hair coat, a different degree of tensioning may be needed for canine TC measurement. This is an important consideration in patients that undergo orthopaedic surgery and rehabilitation, as perceived changes in TC may be due to changes in hair coat alone (i.e. hair regrowth after surgery). This is also important when interpreting previously reported TC measurements.

Several studies report changes in thigh circumference in the months after surgery without specifying the length of hair coat or whether the baseline measurements were taken prior to hair clipping (Slocum and Slocum, 1993, Innes and Barr, 1998, Lauer et al., 2008). Other studies have found significant differences between treatment groups before and after surgical correction, or compared to the contralateral limb. For example, Johnson et al. found a significant difference in TC between CCL-deficient dogs treated with electrical muscle stimulation and a control group at weeks 9 and 13 post-operatively $(30.8 \pm 2.3 \mathrm{~cm}$ vs. $27.2 \pm 2.3 \mathrm{~cm}$ and $30.6 \pm 2.3 \mathrm{~cm}$ vs $27.4 \pm 2.2 \mathrm{~cm}$, respectively), but hair coat length was not controlled between groups (Johnson et al., 1997). Monk et al. demonstrated an increase in TC of $2.75 \pm 0.96 \mathrm{~cm}$ in a surgically corrected limb followed by physical therapy and a decrease in TC of $0.5 \pm 0.28 \mathrm{~cm}$ in surgically corrected limbs without physical therapy, however hair coat length was not described in this paper (Monk et al., 2006). Measurements evaluating 
surgical outcome, or response to treatment, should be performed prior to hair clipping, and only after the hair coat has fully returned. Alternatively the hair coat should be shaved consistently, which seems impractical in a clinical setting. If immediate post-operative changes are to be evaluated, pre-operative measurements should be made after shaving the limb to decrease the effect of hair coat in measurements.

The mid-thigh is the most logical area to be measured when expecting differences in muscle mass due to the bulk of the muscles being located in this area. However, the current descriptions of the pertinent landmarks are imprecise and hence are influenced by the interpretation and palpation skills of each observer. There is no precise way of using anatomic landmarks to determine a repeatable measurement point. The use of laser guidance offers a method of 'marking' the area of interest that is feasible to use even during long-term studies with repeated measurements. While, for the study purposes, we could have marked the area by clipping the fur and using a skin marker, this is not realistic in a clinical setting using client-owned dogs. Hair coat was not clipped in any of the live dogs, so the presence of an intact hair coat may have resulted in overestimation of TC in the second phase of this study. Because we were interested in the reliability of measurement rather than the definitive values, the effect of hair coat on absolute TC in the live dogs was not evaluated. Based upon our study results, the use of a laser-guidance tool may slightly improve inter-observer variability when measuring TC in live dogs. This may be of importance in clinical settings in which multiple observers perform TC measurements. Previous studies have suggested a single observer improves reliability in measurement (Smith et al., 2013, Baker et al., 2010) and our study confirms these findings. However if multiple observers are performing TC measurements on the same patient over time, the use of laser guidance may be indicated to provide a consistent marking of mid-thigh level in an individual patient.

At the patient's first TC measurement, the height of the laser line should be recorded and set accordingly at each subsequent measurement (assuming that the position of the dog and the laser device relative to the wall can be repeated). In this study, intra-observer variability in measurement was similar both with and without laser guidance. This is likely associated with the fact that an individual will use the same palpation methods and interpretation of current guidelines. If TC measurement is to be used as an indirect measure of thigh muscle mass, the current study suggests that an intra-observer variability of at least $1.15 \mathrm{~cm}$ should be assumed. Again, this value in comparison to the cadaveric data calls into question the clinical value of TC measurements.

One limitation of this study was the use of simulated muscle mass changes in cadavers, rather than natural changes in live dogs. This model was developed by the authors of this study to allow making multiple TC measurements across a range of thigh volumes within an individual patient under highly controlled circumstances. This model also decreased the number of cadavers needed to achieve a meaningful dataset representing a range of thigh volumes. The degree of inflation of the submuscular expansion device was chosen to mimic a clinically appreciable increase in muscle mass. While the model was not validated with advanced imaging, we were able to confirm an increased circumference based on palpation as well as visual confirmation during pilot testing. A fluid-filled expansion device was chosen so that leakage could be detected by comparing initial volume to final volume. Under this model it is possible that fluid redistribution within the expansion device contributed to intra- and inter-observer variability. Because the GT is not tensioned to a high degree around the thigh during measurement, this influence was likely minimal.

Comparison of TC between device inflation levels demonstrated that the model produced measureable differences in TC and hence should be considered as a useful model for future studies. Using the described model, a mean TC difference of $1.96 \mathrm{~cm}$ was measured between the lowest $(0 \mathrm{ml}$ total inflation) and highest $(120 \mathrm{ml}$ total inflation) levels of inflation. This cadaveric model of thigh girth expansion is also supported by previous studies, which state that small changes in TC represent significant changes in muscle mass (Doxey, 1987a). A study in men demonstrated that TC changes by only $1.10-1.40 \mathrm{~cm}$ when a workout regimen targeting the thigh muscles is employed (Weiss et al., 2000). Therefore, minimal changes in TC are likely clinically relevant. Ideally a CT scan would have been utilised to confirm true changes in limb circumference under this cadaver model (Thomaes et al., 2012), but this was cost prohibitive for the scope of this study. When 
interpreting our study results, one should also consider that long-haired dogs were used for evaluation of hair clipping; therefore the findings may not be applicable to short-haired dogs. However, given the large difference pre- and post-clipping compared to the difference seen with inflation, the authors suggest that sufficient evidence is presented that hair clipping should be considered when utilising this outcome measure.

In conclusion, we found there to be a significant difference in TC measurements of the same limb with and without hair coat, therefore evaluation of TC following therapeutic intervention should take into account clipping status of hair coat at the time of measurement. We also found that the use of a laser-guidance system nominally (not statistically) improves inter-observer variability for TC measurements. However the high level of variability even when using laser-guidance essentially negates the clinical usefulness of this method. Overall, the high variability in TC measurements made with the GT should be considered when evaluating previous and future studies. Novel techniques for indirect assessment of thigh muscle mass, such as ultrasonographic measurement of TC should be evaluated for the use in canines to provide a more reliable and accurate outcome measure (Mourtzakis and Wischmeyer, 2014).

\section{Abbreviations}

$\mathrm{TC}=$ thigh circumference

CCLD = cranial cruciate ligament disease

$\mathrm{GT}=$ Gulick II tape measure

\section{CONFLICT OF INTEREST}

Disclosure: The authors do not have any conflicts of interest to declare.

Acknowledgments: We would like to thank Keester and her amazing family for the support and contributions - you will never be forgotten.

1. Baker, S. G. et al. (2010) Comparison of four commercial devices to measure limb circumference in dogs. Veterinary and Comparative Orthopaedics and Traumatology, 23 (6), pp. 406-10 http://dx.doi.org/10.3415/VCOT-10-03-0032

2. Berard, A. et al. (2002) Validity of the Leg-O-Meter, an instrument to measure leg circumference. Angiology, 53 (1), pp. 21-8 http://dx.doi.org/10.1177/000331970205300104

3. Berard, A. et al. (1998) Reliability study of the Leg-O-Meter, an improved tape measure device, in patients with chronic venous insufficiency of the leg. Angiology, 49 (3), pp.169-73 http://dx.doi.org/10.1177/000331979804900301

4. Berard, A. and Zuccarelli, F. (2000) Test-retest reliability study of a new improved Leg-O-meter, the Leg-O-meter II, in patients suffering from venous insufficiency of the lower limbs. Angiology, 51, 711-7 http://dx.doi.org/10.1007/978-1-4471-3095-6 135

5. Doxey, G. (1987) Assessing Quadriceps Femoris Muscle Bulk with-Girth Measurements in Subjects with Patellofemoral Pain. Journal of Orthopaedic \& Sports Physical Therapy, 9 (5), pp. 177-83 http://dx.doi.org/10.2519/jospt.1987.9.5.177 
6. Doxey, G. (1987) The Association of Anthropometric Measurements of Thigh Size and B-mode Ultrasound Scanning of Muscle Thickness. Journal of Orthopaedic \& Sports Physical Therapy, 8 (9), pp. 462-8 http://dx.doi.org/10.2519/jospt.1987.8.9.462

7. Eskelinen, E. V. et al. (2012) Canine total knee replacement performed due to osteoarthritis subsequent to distal femur fracture osteosynthesis: two-year objective outcome. Veterinary and Comparative Orthopaedics and Traumatology, 25 (5), pp. 427-32 http://dx.doi.org/10.3415/VCOT11-01-0012

8. Gordon-Evans, W. J.et al. ( 2010) Randomised controlled clinical trial for the use of deracoxib during intense rehabilitation exercises after tibial plateau levelling osteotomy. Veterinary and Comparative Orthopaedics and Traumatology. 23 (5), pp. 332-5 http://dx.doi.org/10.3415/vcot-09$\underline{11-0121}$

9. Gordon-Evans, W. J. et al. (2011) Effect of the use of carprofen in dogs undergoing intense rehabilitation after lateral fabellar suture stabilization. Journal of the American Veterinary Medical Association, 239 (1), pp. 75-80 http://dx.doi.org/10.2460/javma.239.1.75

10. Gordon-Evans, W. J. et al. (2013) Comparison of lateral fabellar suture and tibial plateau leveling osteotomy techniques for treatment of dogs with cranial cruciate ligament disease. Journal of the American Veterinary Medical Association, 243 (5), 675-80 http://dx.doi.org/10.2460/javma.243.5.675

11. Innes, J. F. and Barr, A. R. (1998) Clinical natural history of the postsurgical cruciate deficient canine stifle joint: year 1. Journal of Small Animal Practice, 39 (7), pp. 325-32 http://dx.doi.org/10.1111/j.1748-5827.1998.tb03723.x

12. Jarvela, T. et al. (2002) Simple measurements in assessing muscle performance after an ACL reconstruction. International Journal of Sports Medicine, 23 (3), pp. 196-201 http://dx.doi.org/10.1055/s-2002-23171

13. Johnson, J. M. et al. (1997) Rehabilitation of dogs with surgically treated cranial cruciate ligamentdeficient stifles by use of electrical stimulation of muscles. American Journal of Veterinary Research, 58 (12), pp. 1473-8

14. Kalis, R. H. Liska, W. D. and Jankovits, D. A. 2012. Total hip replacement as a treatment option for capital physeal fractures in dogs and cats. Veterinary Surgery, 41 (1), pp.148-55 http://dx.doi.org/10.1111/j.1532-950X.2011.00919.x

15. Kim, S. E. et al. (2008) Tibial osteotomies for cranial cruciate ligament insufficiency in dogs. Veterinary Surgery, 37 (2), pp. 111-25 http://dx.doi.org/10.1111/j.1532-950X.2007.00361.x

16. Lauer, S. et al. (2008) In vivo comparison of two hinged transarticular external skeletal fixators for multiple ligamentous injuries of the canine stifle. Veterinary and Comparative Orthopaedics and Traumatology, 21 (1), pp. 25-35 http://dx.doi.org/10.3415/VCOT-06-11-0090

17. Millis, D. L. (2004) Assessing and Measuring Outcomes. In: Mills, D.L., Levine, D. and Taylor, R.A. (eds) Canine Rehabilitation \& Physical Therapy, London: WB Saunders. pp. 211-227 http://dx.doi.org/10.1016/B978-0-7216-9555-6.50016-4

18. Millis, D. L. Scroggs, L. and Levine, D. (1999) Variables affecting thigh circumference measurements in dogs. In Blythe, L.J., McCubbin, J.A. and Riebold, T.W. (eds) Proceedings First International Symposium on Rehabilitation and Physical Therapy in Veterinary Medicine, Oregon State University. August 7-11 Oregon: Oregon State University p, 157.

19. Moeller, E. M. et al. (2010) Long-term outcomes of thigh circumference, stifle range-of-motion, and lameness after unilateral tibial plateau levelling osteotomy. Veterinary and Comparative Orthopaedics and Traumatology, 23, pp. 37-42 http://dx.doi.org/10.3415/VCOT-09-04-0043

20. Monk, M. L. Preston, C. A. and McGowan, C. M. (2006) Effects of early intensive postoperative physiotherapy on limb function after tibial plateau leveling osteotomy in dogs with deficiency of the 
cranial cruciate ligament. American Journal of Veterinary Research, 67 (3), pp. 529-36 http://dx.doi.org/10.2460/ajvr.67.3.529

21. Mourtzakis, M. and Wischmeyer, P. (2014) Bedside ultrasound measurement of skeletal muscle. Current Opinion in Clinical Nutrition and Metabolic Care, 17 (5) pp. 389-95 http://dx.doi.org/10.1097/MC0.0000000000000088

22. Slocum, B. and Slocum, T. D. (1993) Tibial plateau leveling osteotomy for repair of cranial cruciate ligament rupture in the canine. Veterinary Clinics of North America: Small Animal Practice, 23 (4), pp. 777-95 http://dx.doi.org/10.1016/S0195-5616(93)50082-7

23. Smith, T. J. (2013) Inter- and intratester reliability of anthropometric assessment of limb circumference in labrador retrievers. Veterinary Surgery, 42 (3), pp. 316-

21 http://dx.doi.org/10.1111/j.1532-950X.2013.01102.x

24. Soderberg, G. L. Ballantyne, B. T. and Kestel, L. L. (1996) Reliability of lower extremity girth measurements after anterior cruciate ligament reconstruction. Physiotherapy Research International, 1 (1), pp. 7-16 http://dx.doi.org/10.1002/pri.43

25. Thomaes, T. et al. (2012) Reliability and validity of the ultrasound technique to measure the rectus femoris muscle diameter in older CAD-patients. BMC Medical Imaging, 12:7 http://dx.doi.org/10.1186/1471-2342-12-7

26. Weiss, L. W., Coney, H. D. and Clark, F. C. (2000) Gross measures of exercise-induced muscular hypertrophy. Journal of Orthopaedic \& Sports Physical Therapy, 30 (3), pp. 143-8 http://dx.doi.org/10.2519/jospt.2000.30.3.143

\section{TABLES \& CHARTS}

Table 1: Phase 1 Cadaveric data - Inter- and intra-observer variability of TC measurement $(\mathrm{in} \mathrm{cm})$ for measurements at all different inflation levels combined

\begin{tabular}{|c|c|c|c|c|c|c|c|c|}
\hline & $\begin{array}{c}\text { Observer } \\
1\end{array}$ & $\begin{array}{c}\text { Observer } \\
2\end{array}$ & $\begin{array}{c}\text { Observer } \\
3\end{array}$ & $\begin{array}{c}\text { Observer } \\
4\end{array}$ & $\begin{array}{c}\text { Inter- } \\
\text { observer } \\
\text { (overall) }\end{array}$ & $\begin{array}{c}\text { Inter- } \\
\text { observer } \\
\text { (hair coat } \\
\text { intact) }\end{array}$ & $\begin{array}{c}\text { Inter- } \\
\text { observer } \\
\text { (hair coat } \\
\text { clipped) }\end{array}$ & $\begin{array}{c}\text { Intra- } \\
\text { observer } \\
\text { (overall) }\end{array}$ \\
\hline $\begin{array}{c}\text { Variability } \pm \text { standard } \\
\text { deviation }(\mathrm{cm})\end{array}$ & $\begin{array}{c}0.88 \\
\pm 0.54\end{array}$ & $\begin{array}{c}0.83 \\
\pm 0.53\end{array}$ & $\begin{array}{c}0.77 \\
\pm 0.52\end{array}$ & $\begin{array}{c}1.45 \\
\pm 1.02\end{array}$ & $\begin{array}{c}2.26 \\
\pm 1.18\end{array}$ & $\begin{array}{c}2.65 \\
\pm .65\end{array}$ & $\begin{array}{c}2.19 \\
\pm 1.19\end{array}$ & $\begin{array}{c}0.90 \\
\pm 0.61\end{array}$ \\
\hline
\end{tabular}

Table 2: Phase 1 Cadaveric data - Comparison of TC measurements made by all observers at different inflation levels and different hair lengths

\begin{tabular}{|c|c|c|c|c|c|c|}
\hline & \multicolumn{2}{|c|}{$\begin{array}{c}\text { Comparison } \\
\text { categories (mls) }\end{array}$} & $\begin{array}{c}\text { Difference } \\
(\mathbf{c m})\end{array}$ & \multicolumn{2}{c|}{$\begin{array}{c}\mathbf{9 5 \%} \\
\text { Confidence } \\
\text { Limits }\end{array}$} & $\begin{array}{c}p- \\
\text { value }\end{array}$ \\
\hline \multirow{2}{*}{$\begin{array}{c}\text { Inflation } \\
\text { level }\end{array}$} & 0 & 120 & 1.96 & 1.76 & 2.16 & $<.0001$ \\
\cline { 2 - 7 } & 0 & 60 & 0.97 & 0.85 & 1.09 & $<.0001$ \\
\cline { 2 - 7 } & 60 & 120 & 0.99 & 0.88 & 1.10 & $<.0001$ \\
\hline Hair length & Intact & Clipped & 3.44 & 2.13 & 4.74 & $<.0001$ \\
\hline
\end{tabular}


Table 3: Phase 2 Live dog data - Pairwise comparisons between observers of difference in TC measurement $(\mathrm{cm})$

\begin{tabular}{|c|c|c|c|c|c|c|}
\hline & \multicolumn{2}{|c|}{ Pairwise comparisons } & Difference (cm) & \multicolumn{2}{c|}{$\begin{array}{c}\text { 95\% Confidence } \\
\text { Limits }\end{array}$} & $p$-value \\
\hline \multirow{4}{*}{$\begin{array}{c}\text { Non } \\
\text { Laser }\end{array}$} & Observer 1 & Observer 2 & 1.53 & -0.84 & 3.90 & 0.21 \\
\cline { 2 - 7 } & Observer 1 & Observer 3 & 2.43 & 0.56 & 4.29 & 0.011 \\
\cline { 2 - 7 } & Observer 1 & Observer 4 & 1.51 & 0.57 & 2.45 & 0.002 \\
\cline { 2 - 7 } & Observer 2 & Observer 3 & 0.90 & 0.06 & 1.73 & 0.036 \\
\cline { 2 - 7 } & Observer 2 & Observer 4 & -0.02 & -2.04 & 2.00 & 0.98 \\
\cline { 2 - 7 } & Observer 3 & Observer 4 & -0.92 & -2.62 & 0.79 & 0.29 \\
\hline \multirow{4}{*}{$\begin{array}{c}\text { Laser } \\
\text { Guided }\end{array}$} & Observer 1 & Observer 2 & 0.67 & -0.54 & 1.88 & 0.28 \\
\cline { 2 - 7 } & Observer 1 & Observer 3 & 0.94 & 0.09 & 1.79 & 0.03 \\
\cline { 2 - 7 } & Observer 1 & Observer 4 & -0.10 & -0.85 & 0.66 & 0.80 \\
\cline { 2 - 7 } & Observer 2 & Observer 3 & 0.27 & -0.55 & 1.10 & 0.52 \\
\cline { 2 - 7 } & Observer 2 & Observer 4 & -0.76 & -1.66 & 0.14 & 0.098 \\
\cline { 2 - 7 } & Observer 3 & Observer 4 & -1.03 & -2.12 & 0.06 & 0.06 \\
\hline
\end{tabular}

Table 4: Phase 2 Live dog data - Variability of TC measurement $(\mathrm{cm})$ with and without laser guidance

\begin{tabular}{|c|c|c|c|c|c|c|c|}
\hline & & Observer 1 & Observer 2 & Observer 3 & Observer 4 & $\begin{array}{c}\text { Inter } \\
\text { observer }\end{array}$ & $\begin{array}{c}\text { Intra- } \\
\text { observer } \\
\text { (all } 4 \\
\text { observers) }\end{array}$ \\
\hline \multirow{3}{*}{$\begin{array}{c}\text { Variability } \\
\pm \text { standard } \\
\text { deviation } \\
(\mathrm{cm})\end{array}$} & $\begin{array}{c}\text { Non } \\
\text { Laser } \\
\text { Guided }\end{array}$ & $1.03 \pm 0.70$ & $1.34 \pm 0.79$ & $1.51 \pm 0.76$ & $0.63 \pm 0.64$ & $4.78 \pm 2.60$ & $1.13 \pm 0.77$ \\
\hline & $\begin{array}{c}\text { Laser } \\
\text { Guided }\end{array}$ & $0.94 \pm 0.50$ & $1.31 \pm 0.58$ & $1.44 \pm 0.79$ & $0.88 \pm 0.67$ & $3.34 \pm 1.09$ & $1.14 \pm 0.66$ \\
\hline & p-value & 0.776 & 0.929 & 0.842 & 0.493 & 0.099 & - \\
\hline
\end{tabular}




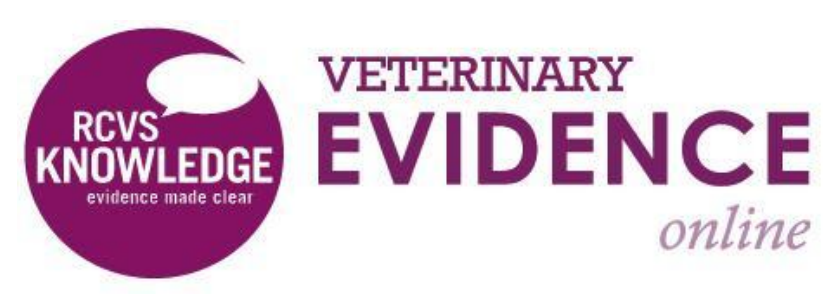

\section{Intellectual Property Rights}

Authors of Knowledge Summaries submitted to RCVS Knowledge for publication will retain copyright in their work, but will be required to grant to RCVS Knowledge an exclusive license of the rights of copyright in the materials including but not limited to the right to publish, re-publish, transmit, sell, distribute and otherwise use the materials in all languages and all media throughout the world, and to license or permit others to do so.

Authors will be required to complete a license for publication form, and will in return retain certain rights as detailed on the form.

Veterinary Evidence and EBVM Network are RCVS Knowledge initiatives. For more information please contact us at editor@veterinaryevidence.org.

RCVS Knowledge is the independent charity associated with the Royal College of Veterinary Surgeons (RCVS). Our ambition is to become a global intermediary for evidence based veterinary knowledge by providing access to information that is of immediate value to practicing veterinary professionals and directly contributes to evidence based clinical decision-making.

\section{www.veterinaryevidence.org}

RCVS Knowledge is a registered Charity No. 230886. Registered as a Company limited by guarantee in England and Wales No. 598443.

Registered Office:

Belgravia House 62-64 Horseferry Road London SW1P 2AF 